NBER WORKING PAPER SERIES

ECONOMICALLY SENSIBLE SOLUTIONS FOR LINEAR RATIONAL EXPECTATIONS MODELS WITH FORWARD AND BACKWARD LOOKING DYNAMIC PROCESSES

Michael Mussa

Working Paper No. 1398

NATIONAL BUREAU OF ECONOMIC RESEARCH 1050 Massachusetts Avenue

Cambridge, MA 02138

July 1984

The research reported here is part of the NBER's research program in International Studies. Any opinions expressed are those of the author and not those of the National Bureau of Economic Research. 
NBER Working Paper \#1398 July 1984

\author{
Economically Sensible Solutions for Linear Rational Expectations Models \\ with Forward and Backward Looking Dynamic Processes
}

\begin{abstract}
Using variants of a modified version of Dornbusch's model of price level and exchange rate dynamics, it is demonstrated that satisfaction of the formal condition for existence of a unigue non-explosive solution of a linear rational expectations model with forward and backward looking dynamic processes (equality of the number of stable roots with the number of independent backward looking processes) does not guarantee the economic sensibility of this solution, even if one accepts the usual arguments for excluding "speculative bubbles" from the solutions of such models. Moreover, satisfaction of the formal condition for existence of an infinity of non-explosive solutions for such rational expectations models (more stable roots than independent backward looking processes) does not assure that any of these solutions is economically sensible.
\end{abstract}

\author{
Michael Mussa \\ Graduate School of Business \\ University of Chicago \\ 1101 East 58th. Street \\ Chicago, Illinois 60637
}

(312) $962-7143$ 


\section{Economically Sensible Solutions for Linear Rational Expectations Models with Forward and Backward Looking Dynamic Processes}

by

Michael Mussa

\section{Introduction}

Rational expectations models applied in monetary and macro economics commonly include both forward looking dynamic processes in which current variables are linked to their expected future values and backward looking dynamic processes in which past values of these variables affect their current behavior. ${ }^{1}$ These models generally admit an infinity of solutions consistent with initial conditions on the predetermined variables. Studies of the characteristics of these solutions have concluded that a unique non-explosive solution exists when the number of stable roots of the dynamic system is equal to the number of independent backward looking dynamic processes, and that an infinity of non-explosive solutions exists when the number of stable roots is greater than the number of independent backward looking processes. ${ }^{2}$ This paper argues that even when there exists one or more non-explosive solutions to such rational expectations models, there is no general presumption that this solution is economically sensible.

This argument is related to, but is different from, the much discussed controversy over the justification for eliminating self-generating speculative bubbles from the solutions of rational expectations models. ${ }^{3}$ Here, we will show that even when the arguments for eliminating such bubbles are accepted as economically justifiable, the formal conditions for existence of non-explosive solutions in rational expectations models do not assure the economic sensibility of such solutions. This will be established by considering variants of a 
generalized version of Dornbusch's (1976) model of exchange rate and price dynamics. In this model, as described in section 2, there is a forward looking dynamic process linking the equilibrium exchange rate and price level to expected future economic conditions, and a backward looking dynamic process linking adjustment of the actual price level to existing deviations from purchasing power parity. The stability properties of solutions of this model are determined by its characteristic roots, $\lambda_{1}$ and $\lambda_{2}$, which are equal, respectively, to the inverse of the absolute value of the interest semi-elasticty of money demand $\left(\lambda_{1}=I / h\right)$ and to minus the speed of response of the price level to deviations from purchasing power parity $\left(\lambda_{2}=-b\right)$.

This identification of characteristic roots with specific parameters is a virtue of this simple model because it enables clear perception of the economic sensibility of various solutions of the model under alternative assumptions about parameter values. When the parameters have economically sensible values ( $h$ and $b$ are both positive), as assumed in section 3 , there is one stable and one unstable root, and the unique non-explosive solution of the model is the economically sensible solution (under the usual argument that excludes speculative bubbles). When $h$ and $b$ are both negative, as assumed in section 4 , the model is economic nonsense, but it still has one stable and one unstable root and, hence, a unique non-explosive solution. This solution, however, is not economically sensible. When $h$ is negative and $b$ is positive, as assumed in section 5 , the model has two stable roots and an infinity of non-explosive solutions. None of these solutions is economically sensible.

These examples establish a general proposition valid in complicated models where the economic interpretation of characteristic roots may be murky. The relationship between the number of stable roots and the number of backward looking processes (or predetermined variables) is not enough to determine the stability properties of economically sensible solutions of the model. 
2. A Model of Price and Exchange Rate Dynamics

Following Dornbusch (1976), consider a small open economy where the condition of money market equilibrium is expressed by

$$
\mathrm{m}=\mathrm{k}+\mathrm{p}-\mathrm{h} \cdot \mathrm{i}
$$

where $\mathrm{m}$ is the logarithm of the nominal money supply, $k$ represents all exogenous factors affecting money demand, $\mathrm{p}$ is the logarithm of the domestic price level, $i$ is the domestic nominal interest rate, and $h$ measures the semi-elasticity of money demand with respect to $i$. The domestic nominal interest rate is linked to the fixed world interest rate, $i *$, through the interest parity condition,

$$
i=i *+D^{e}(e) \text {, }
$$

where $e$ is the logarithm of the exchange rate (defined as the domestic money price of a unit of world money), and $D^{e}(e)$ is the expected rate of change of the exchange rate which is assumed to equal the forward premium on foreign exchange. In the continuous time model, with certainty, $D^{e}(e)$ is the right hand derivative of e with respect to time. In a discrete time model, with uncertainty, $D^{e}(e)=E(e(t+1) ; t)-e(t)$ is the expected forward difference of $e$, where $E(x(s) ; t)$ denotes the expectation of $x(s)$ conditional on information available at time $t$. In either the continuous or the discrete time model, the current exchange rate, $e(t)$, is assumed to be a freely adjustable variable that jumps immediately to the momemtary equilibrium value consistent with (1) and (2), for given values of $m, k, p, i *$ and $D^{e}(e)$.

The domestic price level, in contrast, is assumed to be a slowly adjusting variable whose current value is predetermined by past events. The equilibrium value of $p$ is determined by purchasing power parity to be $e+p^{*}$, where $p^{*}$ is the logarithm of the world price level. Employing the simplifying assumption 
that $\mathrm{p}^{*}=0$, the rule governing the adjustment of $\mathrm{p}$ is assumed to be given by

$$
D(p)=b \cdot(e-p)+D^{e}(e)
$$

where $D($ ) is the forward differential or difference operator. The first term is this price adjustment rule, $b \cdot(e-p)$, describes the backward looling process of adjustment of the sticky domestic price level to the existing state of disequilibrium, as measured by the current deviation from purchasing power parity. The second term in the price adjustment rule, $D^{e}(e)$, prescribes a forward looking adjustment of the price level to expected changes in its equilibrium value. ${ }^{4}$

Under the assumption of rational expectations, $D^{e}(e)$ may be replaced by $D(e)$ in describing the dynamic system that characterizes the expected future paths of the exchange rate and the price level. Solving equation (1) for $i$, and substituting the result into (2), it follows that

$$
D(e)=(1 / h) \cdot[p-w]
$$

where $w=m-k+h \cdot i *$ summarizes the exogenous factors that will influence the course of the exchange rate and the price level in future periods. Using (4) to eliminate $D^{e}(e)$ in (3), it follows that

$$
D(p)=(1 / h) \cdot[p-w]+b \cdot[e-p]
$$

The dynamic system consisting of (4) and (5) determines the expected future paths of $e$ and $p$, starting from an initial time $t=0$, conditional on the expected path of $w(t)$ for $t z 0$ and on the initial price level $p(0)$.

This dynamic system contains a forward looking process arising from the influence of the expected rate of change of the exchange rate on money demand and on the rate of adjustment of the price level, and a backward looking process reflecting adjustment of the price level to deviations from purchasing power parity.' The stability properties of this system are determined by its characteristic roots:

$$
\lambda_{1}=-\mathrm{b} \quad \text { and } \quad \lambda_{2}=1 / \mathrm{h}
$$


The differential equation form of this system has a unique non-explosive solution if $\lambda_{1}$ and $\lambda_{2}$ are opposite in sign, and a one-dimensional infinity of non-explosive solutions if $\lambda_{1}$ and $\lambda_{2}$ both have negative real parts. The difference equation form of the system has a unique non-explosive solution if the modulus of one plus one of the characteristic roots is $>1$ and the modulus of one plus the other characteristic root is < 1 , and a one-dimensional infinity of non-explosive solutions if the modulus of one plus each of the characteristic roots is< 1 .

The next three sections will discuss the economic significance of alternative solutions of the dynamic system (4) and (5) under different assumptions about the parameters $b$ and $h$. Since the results are essentially the same for the differential and difference equation forms of this system, attention will focus on the differential equation form where the solutions can be more easily expressed.

As a preliminary to this discussion, it is important to establish the relationships between the characteristic roots $\lambda_{1}=-b$ and $\lambda_{2}=1 / \mathrm{h}$ and the dynamic processes governing the evolution of the deviation from purchasing power parity, $v=e-p$, and the determination of the equilibrium path of the price level and the exchange rate. Taking the difference between equation (4) and equation (5), it follows that

$$
D(v)=-b \cdot v
$$

Appearance of the characteristic root $\lambda_{1}=-b$ in this dynamic equation indicates the role of this root in controlling the process of adjustment of the deviation from purchasing power parity. The solution of the differential equation form of (7),

$$
v(t)=v(0) \cdot \exp [-b \quad t]
$$

indicates why $\lambda_{1}=-b<0$ is necessary for stability of the process governing adjustment of the deviation from purchasing power parity; clearly, if $\lambda_{1}$ was $>$, 
$v(t)$ would explode at a positive exponential rate whenever $v(0) \neq 0$. This is a sensible stability condition because the process of adjustment of $p$ in response to deviations from purchasing power parity is a backward looking process in which the coefficient b measures the speed of response of the price level to the existing divergence from purchasing power parity.

The relationship between the characteristic root $\lambda_{2}=1 / \mathrm{h}$ and the process governing the behavior of the equilibrium price level and exchange rate is established by considering the process that would govern the common value of $p$ and e if the price adjustment rule (3) were replaced by the assumption that $\mathrm{p}$ is a freely flexible variable that adjusts immediately to maintain purchasing power parity. Defining $q$ as the common equilibrium value of $p$ and $e$, equations (1) and (2) reduce to a single differential or difference equation that is essentially identical to the equation obtained when rational expectations are imposed on Cagan's model of inflationary dynamics; 6

$$
m=k+q-h \cdot[i *+D(q)] .
$$

Rewriting this equation in the form

$$
D(q)=(1 / h) \cdot[q-w]
$$

where $w=m-k+h \cdot i *$, it is apparent that characteristic $\operatorname{root} \lambda_{2}=1 / h$ is the root that is involved in the dynamic process determining the behavior of the equilibrium price level and exchange rate.

From an economic perspective, the dynamic process described by (9) or (10) is forward looking since it arises from an economic relationship in which the equilibrium price level depends on the forward looking expected inflation rate. For such a forward looking process to have an economically sensible solution, the associated characteristic root must be unstable; that is, $\lambda_{2}=1 / \mathrm{h}$ must be positive. When this condition is satisfied, the solution for $q(t)$ that is 
consistent with (9) or (10) can be written as

$$
q(t)=F(t)+C \cdot \exp [(I / h) \cdot t]
$$

where $C$ is an undetermined constant and $F(t)$ is a weighted average of future economic "fundamentals" represented by future w's,

$$
F(t)=\int_{t}^{\infty}(I / h) \cdot w(s) \cdot \exp [(-1 / h) \cdot(s-t)] d s \text {. }
$$

Whenever $C \neq 0$, the term $C \cdot \exp [(1 / h) \cdot t]$ contributes an explosive "speculative bubble" to the path of $q(t)$. This bubble term appears in the general solution of (9) or (10) because it is the solution to the homogenous form of these differential equations (obtained by setting $w=m-k+h \cdot i *$ equal to zero). Since the initial price level is a freely adjustable variable in Cagan's model, it is clear that the speculative bubble can be eliminated from the path of $q(t)$ by choosing $q(0)$ to equal $F(0)$, which amounts to setting $c=0$. Beyond this, it is frequently argued that setting $C=0$ is the economically appropriate choice of $C$. One argument is that this assumption makes the price level depend only on the economic fundamentals that ought to influence its behavior, rather than having $q(t)$ blast off to plus or minus infinity regardless of the behavior of these fundamentals.

For present purposes, it is not essential that this or other arguments for setting $C=0$ be accepted as universally valid. It is essential, however, that the nature of the economic assumption that is made in excluding speculative bubbles from the solution of Cagan's model be clearly understood. It is also essential that this be distinguished from the absence of any economic rationale for assuming that $v(0)=0$ in order to suppress explosive behavior of $v(t)$ when the backward looking adjustment process for deviations from purchasing power parity is unstable due to a neqative value of the adjustment speed, $b$. 
3. The Economically Sensible Solution in the Normal Case

In the normal, economically sensible version of the model of price and exchange rate dynamics, the speed of adjustment of the price level in response to divergences from purchasing power parity is positive (b > 0 ), and the interest semi-elasticity of money demand is negative ( $\mathrm{h}>0$ ). Under these assumptions, the characteristic roots $\lambda_{1}=-b$ and $\lambda_{2}=1 / h$ are real and opposite in sign, implying that the differential equation form of the dynamic system (4) and (5) has a unique non-explosive solution, and a one dimensional infinity of explosive solutions, that are consistent with a given initial price level p(0). The same conclusion applies for the difference equation form of (4) and (5) under the additional economically reasonable assumption that the price level does not over respond to the current divergence from purchasing power parity, as it would in a discrete time model if $b$ were $>1 .^{8}$

Focusing on the differential equation form (4) and (5), the general solution for the paths of $e(t)$ and $p(t)$ can be written as

$$
\begin{aligned}
& e(t)=A \cdot \exp [-b \cdot t]+c \cdot \exp [(1 / h) \cdot t]+F(t) \\
& p(t)=-h b \cdot A \cdot \exp [-b \cdot t]+C \cdot \exp [(1 / h) \cdot t]+F(t)
\end{aligned}
$$

where $F(t)$ is defined by (12) and where the constants $A$ and $C$ must be consistent with the initial condition

$$
-h b \cdot A+C=p(0)-F(0)
$$

By inspection, it is apparent that the first term in these solutions is associated with the characteristic root $\lambda_{1}=-b$ and describes the evolution of the divergence from purchasing power parity, and the second and third terms are associated with the characteristic root $\lambda_{2}=1 / \mathrm{h}$ and describe evolution of the common equilibrium value of the exchange rate and the price level. 
Given the initial price level $p(0)$, there is one degree of freedom in the solutions for $e(t)$ and $p(t)$, the degree of freedom associated with the choice of the constants $A$ and $C$ consistent with the initial condition (15). This degree of freedom allows for a free choice of the initial exchange rate, $e(0)=A+C+F(0)$, for any given $p(0)$. Choice of the initial exchange rate determines the initial divergence from purchasing power parity, $v(0)=e(0)$ - $p(0)$, but does not affect the stability of the process governing the subsequent evolution of this divergence. Specifically, taking the difference between (13) and (14), it follows that $v(t)$ follows the path of exponential decay described by $(8)$, with $v(0)=(1+h b) \cdot A$.

Choice of the initial exchange rate does critically affect the stability of the path of the equilibrium price level and exchange rate, $q(t)=c \cdot e x p[(1 / h) \cdot t]$ $+F(t)$. Specifically, to exclude the explosive term $c \cdot \exp [(1 / h) \cdot t]$ from the equilibrium path of $\mathrm{p}$ and $\mathrm{e}$ it is necessary to set $\mathrm{c}=0$, implying that $\mathrm{A}$ must equal $-(1 / h b) \cdot[p(0)-F(0)]$ and that the initial exchange rate must be

$$
e(0)=F(0)-(1 / h b) \cdot[p(0)-e(0)]
$$

The argument for choosing this initial exchange rate is the same as the argument for choosing $q(t)=F(t)$ as the solution for the equilibrium price level in the rational expectations version of Cagan's model of inflationary dynamics--it is economically sensible that the equilibrium price level should depend on an exponentially weighted average of future w's, but not that it should explode to plus or minus infinity regardless of the behavior of $w$. If one accepts this argument, then the economically sensible solution for the paths of $e(t)$ and $p(t)$ corresponds to the unique non-explosive solution of the dynamic system (4) and (5) that is obtained by setting $c=0$ and $A=-(1 / h b) \cdot[p(0)-F(0)] .^{9}$ 
4. A Nonsense Model with a Unique Non-Explosive Solution

When the speed of adjustment of the price level in response to deviations from purchasing power parity is negative $(b<0)$, the price adjustment rule is not economically sensible and the dynamic process governing the evolution of the divergence from purchasing power parity is not stable. When the interest semi-elasticity of money demand is positive $(h<0)$, there is no economically sensible forward-looking expression for the equilibrium price level in the rational expectations version of Cagan's model of inflationary dynamics. Nevertheless, when the assumption that $b$ is $<0$ is combined with the assumption that $h$ is < 0 , the differential equation form of the dynamic system (4) and (5) has a unique non-explosive solution. This conclusion also applies for the difference equation form of this dynamic system under the additional assumption that $|1 / h|<1$.

Focusing again on the differential equation form of (4) and (5), the general solution for the paths of $e(t)$ and $p(t)$ when $b$ and $h$ are negative can be written as

$$
\begin{aligned}
& e(t)=A \cdot \exp [-b \cdot t]+C \cdot \exp [(1 / h) \cdot t]+B(t) \\
& p(t)=-h b \cdot A \cdot \exp [-b \cdot t]+C \cdot \exp [(1 / h) \cdot t]+B(t)
\end{aligned}
$$

where $B(t)$ is a backward looking weighted sum of past w's,

$$
B(t)=\int_{t}^{0}(1 / h) \cdot w(s) \cdot \exp [(1 / h) \cdot(t-s)] d s
$$

and where the constants $A$ and $C$ must be consistent with the initial condition

$$
-h b \cdot A+C=p(0)-B(0)=p(0) \text {. }
$$

Taking the difference between (17) and (18), it is apparent that the first terms in the solutions for $e(t)$ and $p(t)$ are still the terms that are associated 
with the dynamic process governing the evolution of the divergence from purchasing power parity. Only now, with $b<0$, this process is explosively unstable. The only way to eliminate the effect of this explosive instability on the paths of $e(t)$ and $p(t)$ is by setting $A=0$ and $C=p(0)$. This is equivalent to choosing the initial exchange rate so that there is no initial divergence from purchasing power parity; that is, setting

$$
e(0)=p(0)
$$

This choice of $e(0)$ selects the unique non-explosive solution for $e(t)$ and $p(t)$ out of the class of solutions defined by (17) through (20), just as choice of $e(0)$ in accord with (16) selects the unique non-explosive solution for $e(t)$ and $p(t)$ out of the class of solutions defined by (12) through (15). The argument for imposing (16) when $b$ and $n$ are $>0$, however, does not justify imposing (21) when $b$ and $h$ are $<0$. Imposition of (16) when $b$ and $h$ are $>0$ removes a speculative bubble from the solution path of the equilibrium exchange rate and equilibrium price level on exactly the same basis as the argument for excluding such a speculative bubble from the solution for the price level in Cagan's model. Imposition of (21) when b and $h$ are $<0$ suppresses an explosive, backward looking adjustment process by assuming that there is no initial deviation from purchasing power parity. The economic specification of the model of price and exchange rate dynamics provides no justification for suppressing this genuine source of instability.

The difference between imposing (16) when $b$ and $h$ are positive and imposing (22) when $b$ and $h$ are negative is illustrated in the phase diagrams shown in figures 1 and 2 under the assumption that $w$ is constant at some $\bar{w}$. In both diagrams, the vertical line along which $p=\bar{w}$ shows the combinations of $p$ and $e$ for which $D(e)=(1 / h) \cdot[p-w]=0$; and the line along which $e=p-(1 / h b) \cdot[p-w]$ shows the combinations of $p$ and $e$ for which $D(p)=(1 / h) \cdot[p-w]+b \cdot[e-p]=0$. 


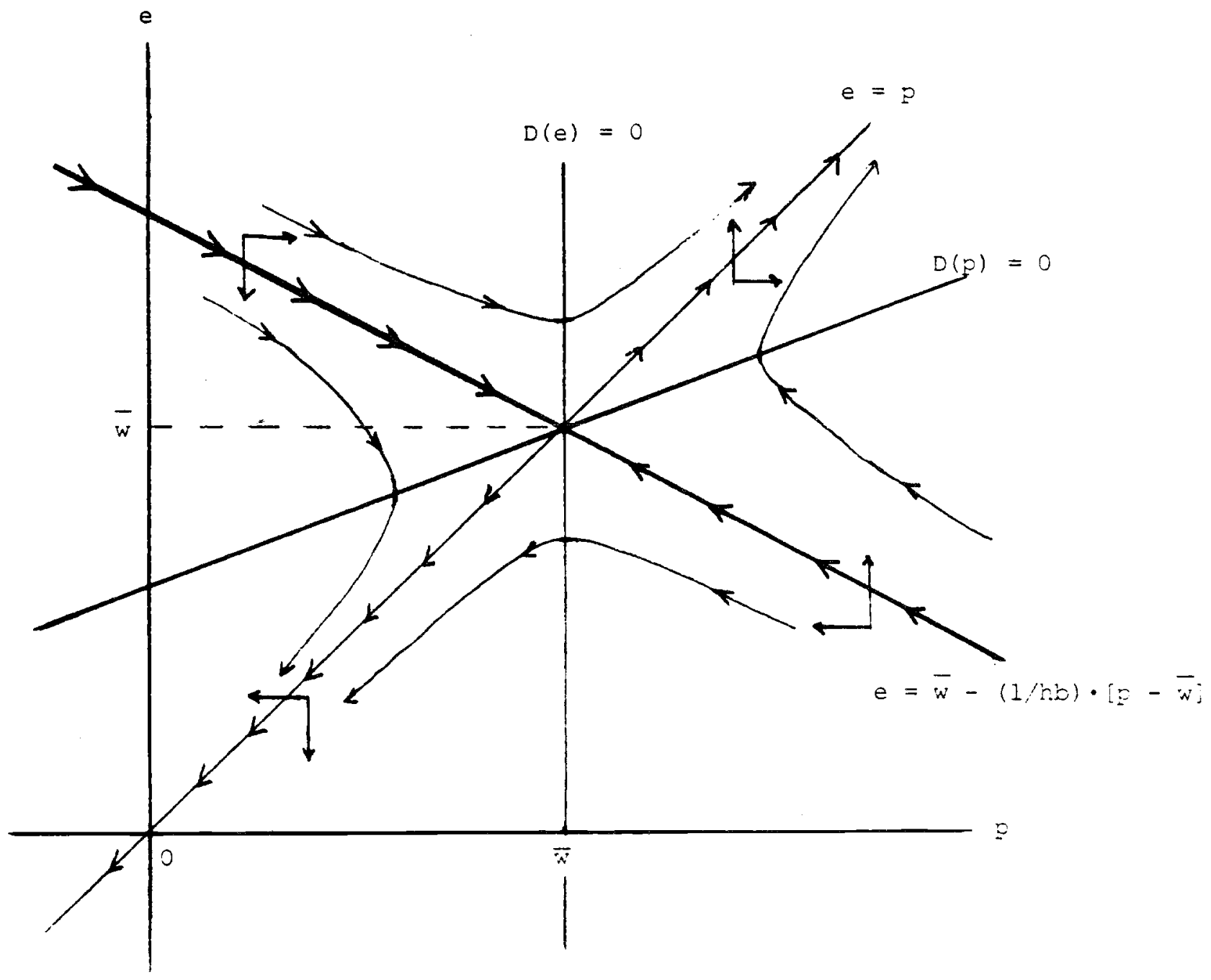

Fig.--1: The Dynamics of $p$ and $e$ with $b>0, h>0$, and $w=\bar{w}$. 


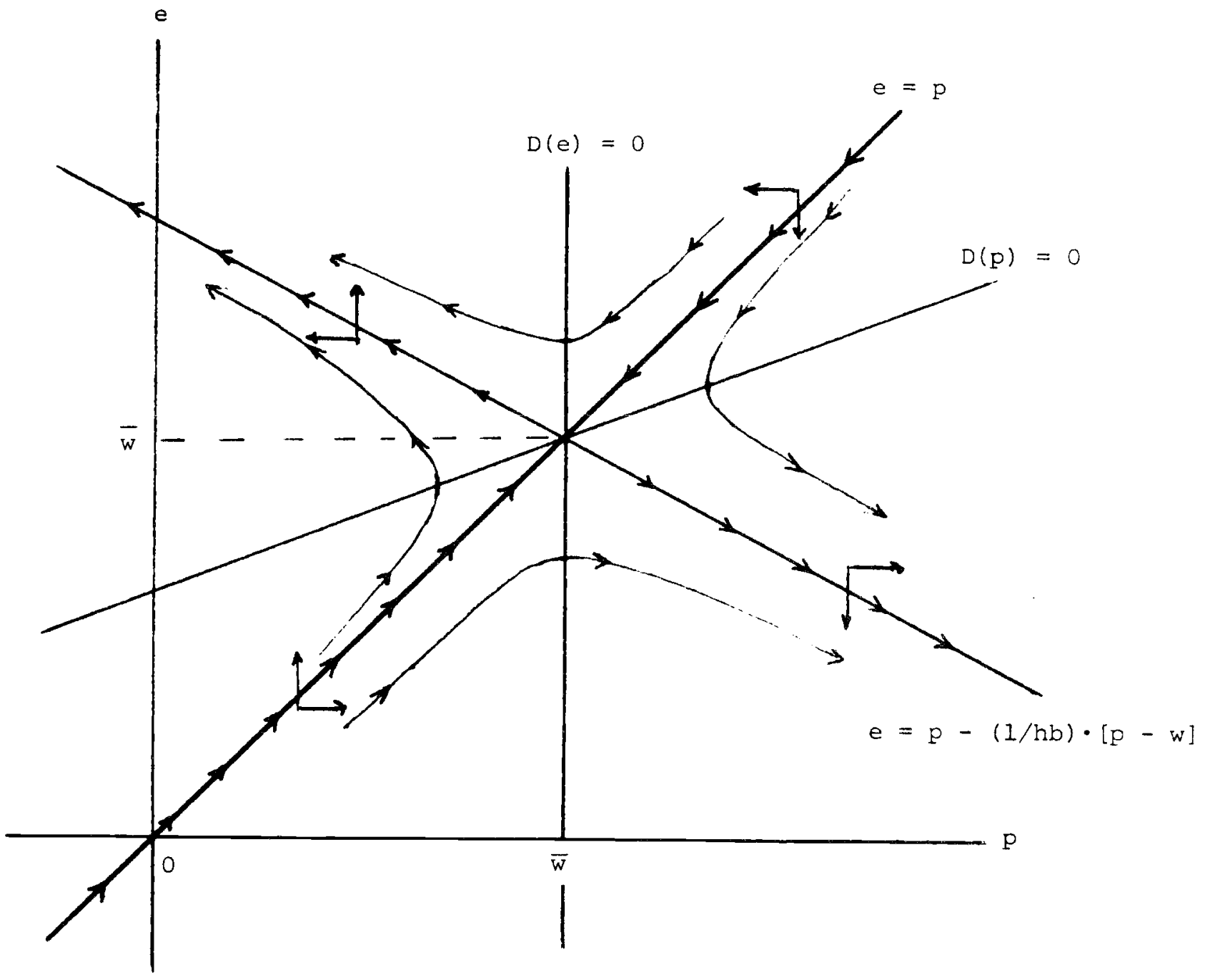

Fig.--2: The Dynamics of $p$ and $e$ with $b<0, h<0$, and $w=\vec{w}$. 
The difference between the two diagrams is in direction of adjustment of $e$ and $p$ for points off of the $D(e)=0$ line and the $D(p)=0$ line. In figure 1 , where $b$ and $h$ are positive, $D(e)$. is $>0$ to the right of the $D(e)=0$ line and $D(e)$ is < 0 to the left of this line, while $D(p)$ is $>0$ above the $D(p)=0$ line and $D(p)$ is $<0$ below this line. Exactly the reverse is true in figure 2 where $b$ and $h$ are negative.

The stable branch in figure $l$ is the line along which $e=\bar{w}-(1 / h b) \cdot[p-\bar{w}]$. Given $p(0)$, when $e(0)$ is set in accord with (16), the initial point $(p(0)$, e(0)) is on this stable branch; and subsequently the point $(p(t), e(t))$ moves toward the point $(\bar{w}, \bar{w})$ at an exponential rate equal to - b. For any other choice of e(0), the divergence from purchasing power parity decays at the exponential rate $-b$, as indicated by convergence of the path of $(p(t), e(t))$ toward the line along which $e=p$, but the common equilibrium component of $p(t)$ and $e(t), q(t)$ $=C \cdot \exp [(1 / h) \cdot t]+\bar{w}$, explodes to plus or minus infinity because of a non-zero value of $C$. Thus, imposition of (16) amounts to choice of the stable branch in figure 1 and is justified by the argument for excluding a speculative bubble from the solution for the equilibrium price level and exchange rate.

In contrast, the stable branch in figure 2 is the line along which $e=p$. Setting $e(0)=p(0)$ places the initial point $(p(0), e(0))$ on this stable branch. Subsequently, the point $(p(t), e(t))$ converges to $(\bar{w}, \bar{w})$ at an exponential rate equal to $1 / h^{10}$ when $e(0) \neq p(0)$, the point $(p(t), e(t))$ converges toward the line that is the stable branch in figure 1 and moves away from the point $(\bar{w}, \bar{w})$ at an exponential rate equal to $-b$. This divergent behavior is not caused by explosive behavior of the common element, $C \cdot \exp [(1 / h) \cdot t]+B(t)$, in the solutions for $p(t)$ and $e(t)$, but rather by explosive behavior of the divergence from purchasing power parity. Thus, choice of the stable branch in figure 2 is not justified by the argument for excluding a speculative bubble from the solution for the equilibrium price level and exchange rate. 
Lack of an adequate justification for choosing the unique non-explosive solution from the class of solutions defined by (17) through (20) is not the only serious problem with these solutions. There is also an important difficulty with the economic interpretation of the term that represents the common equilibrium value of $e(t)$ and $p(t)$ in these solutions; namely, the term

$$
q(t)=C \cdot \exp [(I / h) \cdot t]+B(t)
$$

It is easily verified that (22) provides the general backward looking solution to the differential equation (9) or (10) that governs the behavior of the equilibrium price level in the rational expectations version of Cagan's model of inflationary dynamics. When $h$ is $<0$, it is necessary to use this backward looking solution, rather than the forward looking solution $q(t)=C \cdot \exp [(I / h) \cdot t]$ $+F(t)$, because the integral defining $F(t)$ does not converge, for reasonable assumptions about $w$, when $h$ is $<0$. The mathematical argument for using this backward looking solution, however, does not provide an economic justification for using this solution. In the backward looking solution given in $(22), q(t)$ depends on the past $w^{\prime} s$ between $O$ and $t$, not on future $w^{\prime} s$ between $t$ and $\infty$. This does not make economic sense because the economic condition that determines the equilibrium price level is one in which the current price level is linked to the forward looking expected inflation rate. For this reason, the economically appropriate solution for $q(t)$ is the forward looking solution where $q(t)$ depends on the exponentially weighted average of future w's defined by $F(t)$. From an economic perspective, failure of the integral $F(t)$ to converge when $h$ is $<0$ does not justify using the backward looking solution for $q(t)$. Rather, it indicates that there is no economically sensible expression for the equilibrium price level and exchange rate when the interest semi-elasticity of money demand is positive. 
5. A Case Where Every Solution Is Non-explosive

When the speed of adjustment of the price level in response to divergences from purchasing power parity is positive $(b>0)$, and the interest semi-elasticity of money demand is positive $(h<0)$, the characteristic roots $\lambda_{1}=-b$ and $\lambda_{2}$ $=1 / \mathrm{h}$ are both negative. In this case, every solution of the differential equation form of the dynamic system (4) and (5) is non-explosive. This conclusion also applies to the difference equation form of this system provided that $|1 / \mathrm{h}|<1$.

When $b$ is $>0$ and $h$ is $<0$, the solutions for the paths of $e(t)$ and $p(t)$ are described by equations (17) through (20) of the preceding section. The only difference is that with $b>0$, the first term in the solutions for $e(t)$ and $p(t)$, which involves the factor $A \cdot \exp [-b \cdot t]$, is no longer explosive for non-zero values of $\mathrm{A}$. Since the terms that describe the equilibrium price level and exchange rate, $C \cdot \exp [(1 / h) \cdot t]+B(t)$, are also non-explosive, as they were in the preceding section, it is apparent that for any initial price level $p(0)$, any choice of the initial exchange rate e(0) implies a non-explosive solution for $e(t)$ and $p(t)$. However, because $h$ is $<0$, the backward looking solution $q(t)=C \cdot \exp [(1 / h) \cdot t]+B(t)$, ratier than the forward looking solution $q(t)=c \cdot \exp [(1 / h) \cdot t]+F(t)$, must be used to represent the equilibrium price level and exchange rate. Since this backward looking solution is not consistent with the economic meaning of the forward looking dynamic process (9) or (10) that determines the behavior of the equilibrium price level and exchange rate, none of the solutions for $e(t)$ and $p(t)$ that are described by (17) through (20) is economically sensible.

It is interesting to note that this difficulty with the economic interpretation of the solutions for $e(t)$ and $p(t)$ seems to vanish when $w$ is constant at some $\bar{w}$. In this special case, $B(t)=\bar{w} \cdot[I-\exp ((1 / h) \cdot t)]$. Hence, the 
solutions for $e(t)$ and $p(t)$ given by (17) and (18) can be written as

$$
\begin{aligned}
& e(t)=A \cdot \exp [-b \cdot t]+G \cdot \exp [(1 / h) \cdot t]+\bar{w} \\
& p(t)=-h \cdot b \cdot A \cdot \exp [-b \cdot t]+G \cdot \exp [(1 / h) \cdot t]+\bar{w}
\end{aligned}
$$

where $G=C-\bar{w}$, and where $A$ and $G$ must be consistent with the initial condition

$$
-h b \cdot A+G=p(0)-\bar{w} \text {. }
$$

The behavior of $e(t)$ and $p(t)$ when $w$ is constant at $\bar{w}$ is illustrated in the phase diagram shown in figure 3. The vertical line along which $e=\bar{w}$ shows the combinations of $p$ and e for which $D(e)=(1 / h) \cdot[p-w]=0$. Since $h$ is $<0$, $D(e)$ is < 0 to the right of this line, and $D(e)$ is $>0$ to the left of this line. The line along which $e=p-(1 / h b) \cdot[p-w]$ shows the combinations of $p$ and $e$ for which $D(p)=(1 / h) \cdot[p-w]+b \cdot[e-p]=0$. Since $b$ is $>0, D(p)$ is $>0$ above this line, and $D(p)$ is < 0 below this line. The initial position in the phase diagram is determined by the predetermined initial price level $p(0)$ and the freely chosen initial exchange rate e(0). From this initial position, the point $(p(t), e(t))$ moves in accord with the dynamic laws indicated by the arrows in the phase diagram and converges to the equilibrium point $(\bar{w}, \bar{w})$ with at most a half cycle rotation around this equilibrium.

The behavior of $e(t)$ and $p(t)$ described in this phase diagram (or by equations (23) and (24)) appears to be economically sensible when $\bar{w}$ is regarded as the equilibrium value of the price level and exchange rate. This apparent sensibility, however, conceals a fundamental difficulty. When $h<0$, even with $w$ constant at $\bar{w}$, the forward looking dynamic process that determines the behaviof of the equilibrium price level and exchange rate has no convergent forward looking solution. Hence, while $\bar{w}$ appears to be a sensible measure of the equilibrium price level and exchange rate, there is in fact no such measure. 


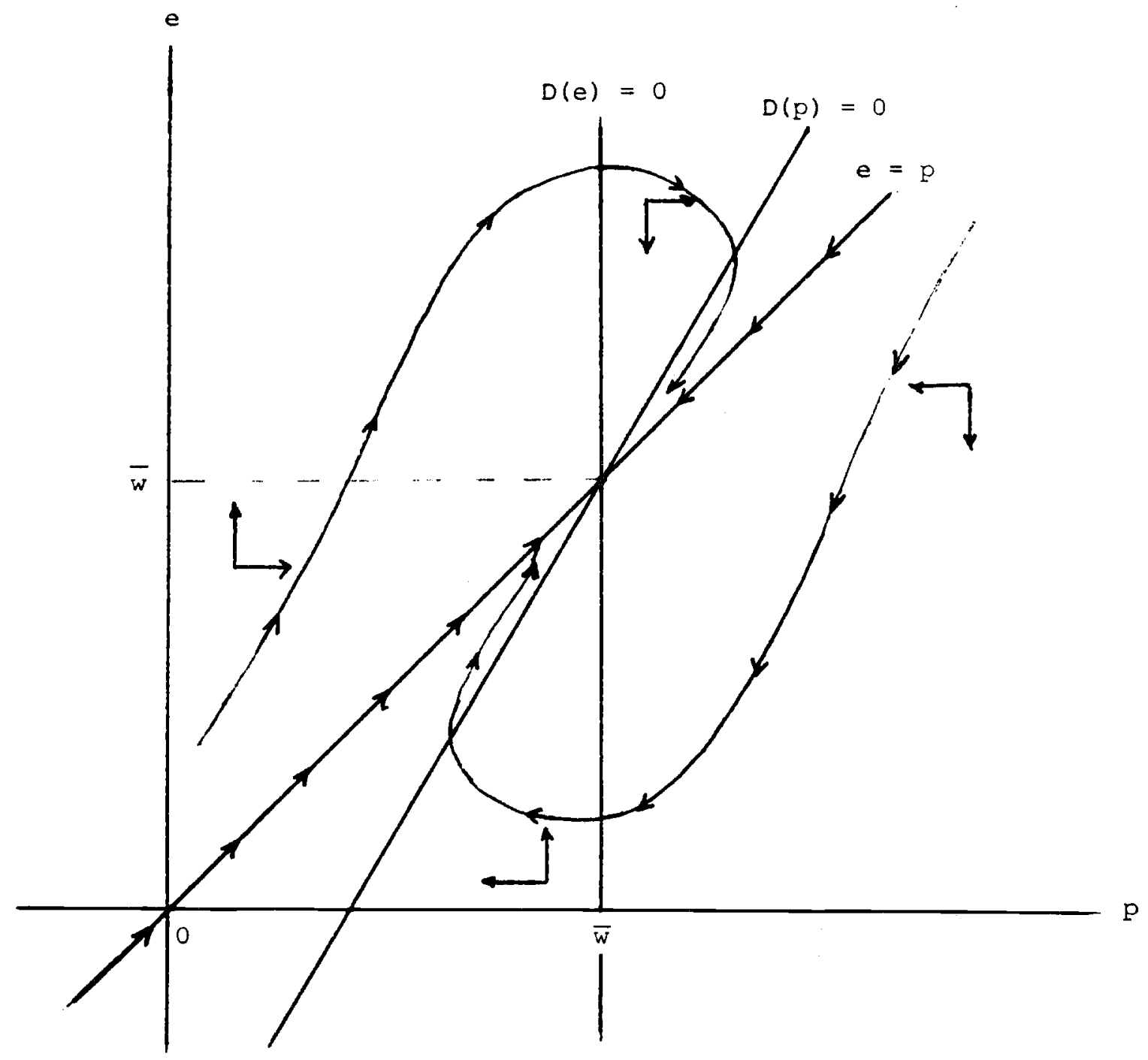

Fig.--3: The Dynamics of $p$ and e with $b>0, h<0$, and $w=\bar{w}$. 


\section{Conclusion}

In this paper, a modified version of Dormbusch's model of price and exchange rate dynamics has been used to establish two general principles concerning solutions to rational expectations models that incorporate both forward and backward looking dynamic processes. First, existence of a unique non-explosive solution for such a model, which is implied by equality of the number of stable characteristic roots with the number of backward looking dynamic processes does not necessarily imply that this solution is economically sensible. Second, existence of a multiplicity of non-explosive solutions for such a model, which is implied by a greater number of stable roots than backward looking dynamic processes, does not necessarily imply that any of these solutions is economically sensible.

The validity of these conclusions is clearly not limited to the relatively simple model that has been used to demonstrate them. The virtue of this model is that there is a clear economic interpretation for each of its characteristic roots and for the dynamic processes with which they are associated. This makes it possible to ascertain, with comparative ease, whether a specific solution for the model is economically sensible. For more complicated models, this task is likely to be both more difficult and more important. In a complicated model with a number of interacting dynamic processes, the economic meaning of specific characteristic roots tends to become obscure, making it difficult to determine whether a particular solution of the model is economically sensible. It is precisely in this situation that comparison of the number of unstable characteristic roots with the number of forward looking dynamic processes is not likely to provide a reliable guide to the economic sensibility of alternative solutions of the model. 
Footnotes

1. This structure is characteristic of models in which prices of goods or assets depend on expected inflation rates or expected rates of capital gain and in which asset stocks or contract wage rates depend on past economic decisions; see, for example, Lucas (1975), Dornbusch (1976), Wilson (1979), Taylor (1980), Buiter and Miller (1981), Blinder and Fischer (1981), Mussa (1981 and 1982), and Basevi and Calzonari (1984)

2. The formal conditions for the existence of non-explosive solutions to linear rational expectations models with forward and backward looking processes are examined in Blanchard and Kahn (1980) and Buiter (1982a). Also see Calvo (1978 and 1979), Blanchard (1979), Burmeister (1980), Buiter (1982b), Lipton, et. al. (1980), Dixit (1980), and Taylor (1977).

3. An insightful discussion of this controversy and references to the relevant literature are given in McCallum (1983).

4. The price adjustment rule (3) is somewhat different from the rule used by Dornbusch (1976), but it does incorporate in the term b (e - p) the same essential mechanism of correction for existing disequilibrium as appears in Dornbusch's price adjustment rule. The additional term $D^{e}(e)$ in the price adjustment rule (3) can be justified economically on the grounds discussed by Mussa (1981 and 1982). Inclusion of this term also allows for very convenient expressions of the characteristic roots of the dynamic system implied by the model of price and exchange rate dynamics in terms of the parameters $b$ and $h$.

5. It is preferable to speak of the distinction between forward looking and backward looking dynamic processes, rather than forward looking and backward looking variables, or freely flexible and predetermined variables. In the present model, the price level is predetermined, but its dynamic behavior is 
influenced by both forward and backward looking dynamic processes. The exchange rate is a freely flexible variable, but at least in the economically sensible solution of the model of section 3 , the initial exchange rate depends on the predetermined price level. Moreover, the model of price and exchange rate dynamics (or any other linear dynamic model) can always be written in terms of independent linear combinations of the original variables. This rewriting can usually be done so that no variable is wholly controlled by either a forward or a backward looking dynamic process, and so that no variable is either wholly predetermined or completely flexible. Such a rewriting, however, does not alter the mathematical or economic properties of the model.

6. For analysis of Cagan's model under rational expectations, see especially Sargent and Wallace (1973a and 1973b). Also see Mussa (1975 and 1978).

7. In some cases, it is possible to eliminate the explosive bubble on the grounds that it is inconsistent with maximizing behavior of individual economic agents, but this is not possible in all such cases even when the theoretical model deals explicitly with individual maximizing behavior; see Brock (1974 and 1975), Brock and Scheinkman (1980), Calvo (1979) and Scheinkman (1980). In the case of the German hyperinflation, the empirical evidence examined by Flood and Garber (1980) appears to justify exclusion of an explosive bubble from the solution for the path of the price level.

8. In a discrete time model, if we allow the length of the period to become shorter and shorter, the constraint that $\mathrm{b}$ must be $<1$ can be made consistent with an arbitrarily speed of adjustment of the price level, in real time, in response to deviations from purchasing power parity. Thus, there is no important difference 
between the constraint that needs to be imposed on this adjustment speed in the discrete and continuous time versions of the model of price and exchange rate dynamics.

9. This is essentially the same as the solution that Dornbusch uses in his analysis. As Dornbusch explains, when the price level is below its long run equilibrium level, the interest rate must be below its long run equilibrium level in order to maintain momentary equilibrium in the money market. To maintain interest parity with this low interest rate, there must be the expectation of a decrease in the exchange rate. For this expectation to be rational, the current exchange rate must be above its long run equilibrium level by exactly the right amount, given the speed of convergence of the exchange rate toward its long run equilibrium level.

10. No sensible economic explanation can be given for why the speed of convergence of the price level and the exchange rate toward the common value $\bar{w}$ should depend on the inverse of the interest semi-elasticity of money demand. or, even more peculiar, why this speed of convergence should be independent of the coefficient $b$ in the price adjustment rule. 


\section{References}

Basevi, Giorgio and Calzonari, Michele, 1982, "Multilateral Exchange Rate Determination," a paper presented at the Bellagio Conferecne on Exchange Rates: Theory and Practice.

Blanchard, Olivier, 1979, "Backward and Forward Looking Solutions for Economies with Rational Expectations," American Economic Review, 69, no. 1 $(\operatorname{March}), 114-118$

Blanchard, Olivier and Kahn, Charles, 1980, "The Solution of Linear Difference Equation Models under Rational Expectations," Econometrica, 48, no. 5 (July), 1305-1311.

Blinder, Alan and Fischer, Stanley, 1981, "Inventories, Rational Expectations and the Business Cycle," Journal of Monetary Economics, 8, no. 3 (November), 277-304.

Brock, William, 1974, "Money and Growth: The Case of Long-Run Perfect Foresight," International Economic Review, 15, no. (October), $750-777$. ' 1975, "A Simple Perfect Foresight Monetary Model," Journal of Monetary Economics, 1, no. 2 (April), 133-150. Brock, William, and Scheinkman, Jose, 1980, "Some Remarks on Monetary Policy in an Overlapping Generations Model," in John Kareken and Neil Wallace, eds., Models of Monetary Economies. Minneapolis, Federal Reserve Bank of Minneapolis, 211-232.

Buiter, willem, 1982a, "Saddle Point Problems in Continuous Time Rational Expectations Models: A General Method and some Macroeconomic Examples," NBER Technical Working Paper No. 20. (February).

' 1982b, "Predetermined and Non-Predetermined Variables in Rational Expectations Models," Economic Letters, 10, 49-54. 
Buiter, Willem, and Miller, J. Marcus, 1981, "Monetary Policy and

International Competitiveness: The Problems of Adjustment," Oxford

Economic Papers, 143-175.

Burmeister, Edwin, 1980, "On Some Conceptual Issues in Rational Expectations Modeling," Journal of Money, Credit and Banking, 12, no. 4, Part II

(November), 800-816.

Calvo, Guillermo, 1978, "On the Indeterminacy of Interest Rates and Wages with Perfect Foresight," Journal of Economic Theory, 19, no. 2 (December), 321327.

, 1979, "On Models of Money and Perfect Foresight," International

Economic Review, 20, no. 1 (February), 83-103.

Dixit, Avinash, 1980, "A Solution Technique for Rational Expectations Models

with Applications to Exchange Rate and Interest Rate Determination,"

Discussion Paper, Department of Economics, University of Warwick

(November).

Dornbusch, Rudiger, 1976, "Expectations and Exchange Rate Dynamics," Journal of Political Economy, 84, no. 6 (December), $1161-1176$.

Flood, Robert and Garber, Peter, 1980, "Market Fundamentals Versus Price Level Bubbles: The First Tests," Journal of Political Economy, 88, no. 4 (August), 745-770.

Lipton, David, et. al., 1980, "Multiple Shouting in Rational Expectations Models," NBER Technical Working Paper No. 3 (August).

Lucas, Robert E., 1975, "An Equilibrium Model of the Business Cycle," Journal of Political Economy, 83, no. 6 (December), $1113-1144$. McCallum, Bennett, 1983, "On Non-Uniqueness in Rational Expectations Models," Journal of Monetary Economics, 11, no. 2 (March), 139-168. 
Mussa, Michael, 1975, "Adaptive and Regressive Expectations in a Rational Model of the Inflationary Process," Journal of Monetary Economics, 1, no. 4 (October), 423-442. , 1978, "On the Inherent Stability of Rationally Adaptive Expectations, Journal of Monetary Economics, 4, no. 3 (July), 307-313. , 1981, "Sticky Prices and Disequilibrium Adjustment in a Rational Model of the Inflationary Process," American Economic Review, 71, no. 5 (December), 1020-1027. , 1982, "A Model of Exchange Rate Dynamics," Journal of Political Economy, 90, no. 1 (February), 74-104. Sargent, Thomas and Wallace, Neil, 1973a, "Rational Expectations and the Dynamics of Hyperinflation," International Economic Review, 14, no. 2 (June), 328-350. ' 1973b, "The Stability of Models of Money and Growth with Perfect Foresight," Econometrica, 41, no. 6 (November), 1043-1048. Scheinkman, Jose, 1980, "Discussion," in John Kareken and Neil Wallace, eds., Models of Monetary Economies. Minneapolis: Federal Reserve Bank of Minneapolis, 91-96.

Taylor, John B., 1977, "Conditions for Unique Solutions in Stochastic Macroeconomic Models with Rational Expectations," Econometrica, 45, no. (September), 1377-1385. ' 1980, "Aggregate Dynamics and Staggered Contracts," Journal of Political Economy, 88, no. 1 (February), 1-23. 